\title{
Implementing Evidence-Based Task Strengthening Strategies for Hypertension Control within Ghana's Community-Based Health Planning Services: A Concept Mapping Analysis
}

Solomon Nyame ( $\sim$ solomon.nyame@kintampo-hrc.org)

Kintampo Health Research Centre https://orcid.org/0000-0003-0419-7237

Juliet Iwelunmor

College for Public Health and Social Justice, Department of Behavioral Science and Health Education, Saint Louis University

Kwaku Poku Asante

Kintampo Health Research Centre

Joyce Gyamfi

Division of Health and Behaviour, Department of Population Health, NYU Langone Medical Center, New York University

Kwame Adjei

Kintampo Health Research Centre

Kingsley Apusiga

Kwame Nkrumah University of Science and Technology

Deborah Onakomaiya

Division of Health and Behaviour, Department of Population Health, NYU Langone Medical Center, New

York University

Kezia Gladys Amaning Adjei

Kwame Nkrumah University of Science and Technology

Angela Aifah

Division of Health and Behavior, Department of Population Health, NYU Langone Medical Center, New York University

Jacob Plange-Rhule

Kwame Nkrumah University of Science and Technology

Gbenga Ogedegbe

Division of Health and Behaviour, Department of Population Health, NYU Langone Medical Center, New York University 
Keywords: task-strengthening strategy for hypertension control (TASSH), Ghana's Health Services (GHS), Community-based Health and Planning Services (CHPS)

Posted Date: June 30th, 2021

DOI: https://doi.org/10.21203/rs.3.rs-155327/v2

License: (c) (1) This work is licensed under a Creative Commons Attribution 4.0 International License. Read Full License 


\section{Abstract}

Background: As efforts are made to systematically implement an evidence-based task-strengthening strategy for hypertension control (TASSH) in Ghana, understanding their content can help national, regional and district health stakeholders within Ghana's Health Services (GHS) identify strategies for enhancing intervention uptake and sustainability in their local context. This study sought to describe national, regional and district health stakeholders' perspectives and characterize the array of strategies needed to enhance the uptake of evidence-based TASSH within Ghana's Community-based Health and Planning Services (CHPS) zones.

Methods: This qualitative study was conducted among national, regional and district health stakeholders within GHS serving patients who utilize CHPS zones. The CHPS initiative is Ghana's flagship strategy for achieving universal health coverage. Semi-structured interviews were conducted with 22 national, regional and district health stakeholders identified via purposive sampling. Interviews were conducted in 2018. Data analysis was conducted between July 2019 and December 2019. Data was analyzed using group concept mapping systems to identify major themes and subthemes.

Results: About $68 \%$ of participants were male with mean age of 40 years and mean years of experience providing hypertension-related care within GHS of 9 years. A conceptual map emerged, consisting of 46 strategies needed for implementing evidence-based TASSH, organized into 6 clusters: 1) Referral Systems; 2) Availability of Equipment; 3) Protocols and Guidelines; 4) Capacity Building/Training; 5) Policy Reform, and 6) Technical Support and Supervision. Availability of equipment was rated as the most important strategy (mean 4.80 out of 5) needed to implement evidence-based TASSH, while Capacity Building/Training was rated as the most feasible strategy (mean 4.20 out of 5 ) to address. Although important (mean 4.40 out of 5), Policy reform was rated as the least important and feasible strategy to address.

Conclusions: These findings demonstrate strategies that can help inform future interventions focused on the adoption and sustainability of evidence-based TASSH within Ghana's CHPS zones. Also, national, regional and district health stakeholders can support healthcare workers by facilitating access to equipment and strategies for enhancing capacity and training with implementing evidence-based taskshifting hypertension interventions in Ghana.

\section{Background}

According to the World Health Organization (WHO), 75\% of deaths in Sub-Saharan Africa (SSA) will be attributable to hypertension (HTN) by the year 2020 [1]. Thus, interventions targeted at blood pressure (BP) control are vital to reducing hypertension-related morbidity and mortality [2, 3]. However, socioeconomic barriers, lack of insurance coverage, uncoordinated care, and shortage of physicians limit the capacity of SSA countries to implement Cardiovascular Disease (CVD) prevention at the primary care level [4-9]. Of these, systems-level barriers have a more adverse impact on health outcomes [10]. Thus, 
poor access to care and limited availability of laboratory tests are major barriers limiting the capacity of countries in SSA to manage a chronic disease like HTN [5].

The situation in SSA is compounded by the acute shortage of health workers available to implement CVD prevention at the primary care level. For example, 36 of the 57 countries that the WHO identified with a critical shortage of health workers are in SSA [11]. Although SSA harbors $11 \%$ of the world's population, it bears over $24 \%$ of the world's disease burden and only has $3 \%$ of the global health workforce [11]. Shortage of physicians is a major barrier to HTN control in Ghana, whereby in 2017 , the density of doctors, nurses and midwives were 2.65 per 1000 population $[12,13]$. The shortage of healthcare providers hinders Ghana's capacity to control HTN at the primary care level, where the majority of patients receive care [14]. Thus, there is a need to implement and integrate bold innovative strategies into already existing infrastructure within the Ghana Health Service such as the successful Community-Based Health Planning and Services programme (CHPS) [15]. The CHPS concept is Ghana's key strategy for changing the focus of primary health care from the district and sub-district health facilities to community and doorstep locations $[15,16]$.

Studies have demonstrated the effectiveness of using the evidence-based Task- strengthening Strategy for high blood pressure control (TASSH) model tailored to the needs of community health nurses to deliver hypertension care $[17,18]$. In 2015, we established in a cluster RCT in 32 district hospitals and community health centers in Ashanti Region, Ghana, that an evidence-based Task-Shifting Strategy for HTN Control (TASSH) based on the WHO Cardiovascular Risk Package and the provision of health insurance coverage (HIC) to patients, delivered by community health nurses (CHNs) led to a $20.4 \mathrm{mmHg}$ mean reduction in systolic blood pressure (SBP) for TASSH + HIC group and $16.8 \mathrm{mmHg}$ mean reduction in SBP for HIC group at 12 months [17].

Understanding how to systematically implement evidence-based TASSH interventions in Ghana, and its content can help national, regional and district health stakeholders within Ghana's Health Services. This study utilized Group Concept Mapping (GCM) to examine factors likely to influence the adoption of TASSH within the CHPS zones. Group Concept Mapping (GCM) is a mixed-methods design that engages participants through the research process in well-structured qualitative and quantitative methods [19-24]. The approach allows researchers to capture, organize, and rate conceptual data from individual and groups. It as well creates a meaningful conceptualization of the issues under investigation [20-23]. We thoroughly used GCM to evaluate national, regional and district health stakeholders' perspectives and characterize the array of strategies needed to enhance uptake of evidence-based TASSH within Ghana's Community-based Health and Planning Services (CHPS) zones.

\section{Methods}

\section{Participants}


Semi-structured interviews were conducted with 22 national, regional and district health stakeholders identified via purposive sampling. The stakeholders who participated are national policymakers with the Ghana Health Service and Ministry of Health, Brong Ahafo regional policy implementors with the Ghana Health Service, as well as district-level policy implementors within the Kintampo North and South districts. Participants were identified through the steering committee group formed to guide the implementation of community-based management of hypertension. Information on context as well as ways to improve practice capacity for the uptake of the study intervention was collected from the identified stakeholders. All procedures as described were approved by three independent ethics review boards. These ethics review boards are the Ghana Health Service Ethics Review Committee, the Committee on Human Research Publication and Ethics, Kwame Nkrumah University of Science and Technology as well as the Kintampo Health Research Centre Institutional Ethics Committee. Written informed consent was obtained from all participants.

\section{Procedure}

To better understand the context and identify practice capacity for the uptake of TASSH in CHPS compounds the study utilized the six steps of GCM $[25,26]$ which are described below:

1) Preparation Stage: During this phase, stakeholders were pre-identified, and a focus prompt to guide the brainstorming exercise was created. The focus prompt asked, "What do CHPS compound nurses need to successfully use TASSH ("identify, treat, refer") for hypertension control?" This exercise was conducted face-to-face, so that the project's goal was explained to participants before the concept-mapping exercise commenced [26].

2) Generation Stage (brainstorming stage): In this phase, key stakeholders took part in the preparation stage and brainstormed a set of statements related to the focus prompt. In response to the focus prompt, the stakeholders identified at various levels of leadership provided statements. Each respondent's demographic information was gathered [26].

3) Structuring Stage (sorting and rating): In this phase, each participant sorted the statements into piles based on similarity and then rated each statement based on its perceived importance (i.e. how important is this strategy) and feasibility (i.e. how feasible is this strategy) of the statements generated in the previous phase.

After the participants sorted the statements, they rated each statement on a 4-point Likert scale based on importance and feasibility.

4) Representation Stage (concept-mapping data analysis): The concept mapping data analyses were conducted using the Concept System software. The sorted data was used to conduct multidimensional scaling (MDS) analysis with a two-dimensional solution as well as hierarchical cluster analysis [27, 28]. The MDS analysis was based on the measurement model that assumes that the relative similarity of 
objects can be represented in terms of the relative distance between pairs of points [28]. To indicate the goodness of fit, a "stress value" of the point map was developed to determine how well the MDS solution maps the original data [28]. With concept mapping, a lower stress value indicates a better fit and reflects a stronger relationship between the optimal and actual configurations [27, 28]. Furthermore, hierarchical cluster analysis ( $\mathrm{HCA}$ ) was conducted using the two-dimensional $x-y$ coordinate data obtained from the MDS analysis as input and applying Ward's algorithm as the basis for defining clusters $[28,29]$. This approach forces the cluster analyses to partition the MDS configuration into non-overlapping clusters in two-dimensional space [27, 29]. This technique also grouped the outcome statements on each map such that statements placed in the same cluster were located at contiguous areas of the map.[27, 29] The resulting output was a "cluster map" which revealed how the statements generated, as represented by points, are grouped [28]. Also, mean ratings for importance and feasibility, respectively, of each statement and cluster were estimated. A pattern-matching graph was used to graphically show the agreement in mean cluster ratings between what is feasible versus what is important across the various stakeholders [22]. Another output from the pattern matching graph is Pearson's product-moment correlation coefficient ( $r$ ) which is an indication of the strength of the relationship between the variables of interest (in our case feasibility and importance) [22]. Also, the t-test is often used to compare the mean ratings.[22] Lastly, a two-dimensional Go-zone graph was generated to visually plot the ratings of each statement on both importance and feasibility concurrently.[22] This graph puts statements that were rated above the mean for both importance and feasibility positioned in the top right quadrant (Go-zone).[22] This shows high priority statements (activities/actions) that will facilitate the uptake of evidence-based task-shifting strategies for hypertension control in Ghana.

5) Interpretation Stage: Interpretation of results is a real-time, participatory process where stakeholders interacted with the ideas generated.[22] This activity included examining the cluster maps to determine which clusters of statements were rated most important to the focus statement, examining the patternmatching to determine key areas to target based on high ratings, and examining the go-zones to determine the area of most importance for each stakeholder group[27]. This activity ensured that all participants were involved in explaining the visual maps and graphs.

6) Utilization Stage: In this stage, we worked with the stakeholders to determine the best ways to use the maps and reports produced by the GCM procedures. The concept mapping output includes creating priority areas for improving practice capacity for TASSH uptake which in turn served as the basis for planning the implementation of TASSH at CHPS compounds.

\section{Results}

\section{Characteristics of participants}

Table 1 describes the demographic characteristics of the participants. Of the 22 participants $68 \%$ were male, with a mean (SD) age of 40 (7.5) years, and had on average 9 (SD: 7.04) years of experience providing hypertension-related care within the Ghana Health Service. 
Table 1

Demographic characteristics of participants

\begin{tabular}{|c|c|}
\hline \multirow[t]{2}{*}{ Variable } & $\mathrm{n}(\%)$ \\
\hline & $N=22$ \\
\hline \multicolumn{2}{|l|}{ Sex } \\
\hline Male & $15(68)$ \\
\hline Female & $7(32)$ \\
\hline \multicolumn{2}{|l|}{ Age } \\
\hline $18-34$ & $1(4.6)$ \\
\hline $35-44$ & $14(63.6)$ \\
\hline $45-54$ & $2(9.1)$ \\
\hline $55-64$ & $5(22.7)$ \\
\hline \multicolumn{2}{|c|}{ mean (SD) age, 40 (7.5) years } \\
\hline \multicolumn{2}{|l|}{ Educational Level } \\
\hline Bachelor's Degree & $11(50.0)$ \\
\hline Master's Degree & $6(27.3)$ \\
\hline Doctorate Degree & $5(22.7)$ \\
\hline mean $(S D)$ of $9(7.0$ & rtension-related care within GHS \\
\hline
\end{tabular}

A six-cluster map was considered to be the most suitable with statements within each cluster rationally belonging together (Fig. 1 and Table 2). The conceptual map consisted of 46 strategies needed for implementing evidence-based TASSH, organized into 6 clusters: 1) Referral Systems; 2) Availability of Equipment; 3) Protocols and Guidelines; 4) Capacity Building/Training; 5) Policy Reform, and 6) Technical Support and Supervision. The number of statements in the clusters ranged from 3 (referral system) to 15 (Capacity building/training) 
Table 2

Six themes with corresponding statements

Themes

1. Referral System

2. Availability of equipment

\section{Statements}

1. Availability of resources such as referral materials and home visit bags

38. Available of strategies for a referral to care

2. Strengthening of the referral process from lower to high-level health facilities

20. Improve resources and tools essential for hypertension management at the CHPS Zones

5. Access to reliable transport services or motorbikes for home visits

13. Facilitate movement around the CHPS zone by providing bicycles and motorbikes

19. Investigation tools at the CHPS zones such as a glucometer, glucometer strips, lancets, alcohol swaps/cotton

7. Make Blood Pressure instrument/sphygmomanometer readily available at the CHPS Zones

21. Availability of Sphygmomanometers for routine screening by all health workers

24. Availability and maintenance of Blood Pressure monitor and the need to periodically calibrate apparatus

17. Provision of equipment at the CHPS Zones

25. Provide stethoscope and weighing scale for measuring the patient's $\mathrm{BMI}$

32. Provision of Stationaries for record-keeping at the CHPS zones

3. Protocol and guidelines

37. Available protocol to provide guidelines

33. Availability of materials such as research protocols

18. Access to risk assessment tools

41. Teaching materials to create more awareness of hypertension, such as posters, pamphlets, charts

31. Provide easy guidelines for the health workers to appropriately identify, treat or refer the hypertensive cases

4. Capacity building/training
4. Promote capacity building initiatives at the CHPS Zones

11. Training nurses on referral pathways at the CHPS Zones 
29. Providing training to nurses in the proper methods for treating mildto-moderate hypertensive cases

39. Train nurses at the CHPS Zones to manage hypertensive patients

43. Adequate and routine training on the diagnosis and management of HTN

3. Creating awareness of accurate blood pressure measurement

8. Train health workers at the sub-district and district health facilities on TASSH Strategies

9. Refresher training on identification, counselling and referral of hypertensive cases

10. Strategies to strengthen and build logistics capacity for data collection

14. On-going assessment of the nurse's ability and capacity to treat hypertension at the CHPS Zones

16. Regular and Intensive training for the CHPS Zone health workers

22. Provide on the job training to the CHPS Zone health workers

27. Train health workers at the CHPS Zones on how to correctly take blood pressure measurements

28. Providing broad knowledge on how to correctly identify hypertensive cases

44. Means of transportation at the CHPS Zones

5. Policy reform

35. Availability of the Minimum required hypertensive medication at the CHPS Zone

\section{Policy reform at the CHPS Zones}

12. Include TASSH strategies in the CHPS policy and implementation

42. Presence of a medical officer or clinician supervising the CHPS compound to enhance optimal HTN care and control

46. Presence of a physician assistant at the CHPS zone to manage hypertensive cases

36. Improve access to hypertensive medications

6. Provide technical support and supervision
23. Provision of incentives that will yield community buy-in

34. Provide technical support to CHPS zone nurses from higher-level health facilities

15. Availability of case management guidelines 


\begin{tabular}{|c|c|}
\hline \multirow[t]{5}{*}{ Themes } & Statements \\
\hline & $\begin{array}{l}\text { 26. Work with key leaders to enhance and support TASSH uptake and } \\
\text { implementation within CHPS zones. }\end{array}$ \\
\hline & $\begin{array}{l}\text { 30. Periodically monitor and supervise health workers at the CHPS } \\
\text { Zones }\end{array}$ \\
\hline & $\begin{array}{l}\text { 40. Provide on-site supervision to nurses at the CHPS zones from the } \\
\text { subdistrict and the district hospitals }\end{array}$ \\
\hline & $\begin{array}{l}\text { 45. Offer training to leaders for effective supervision of performance at } \\
\text { the CHPS Zones }\end{array}$ \\
\hline
\end{tabular}

Figure 2 shows the mean important as well as feasibility strategy. Availability of equipment was rated as the most important strategy (mean 4.80 out of 5 ) needed to implement evidence-based TASSH, while Capacity Building/Training was rated as the most feasible strategy (mean 4.20 out of 5 ) to address. Although important (mean 4.40 out of 5), Policy reform was rated as the least feasible and important strategy to address. The stress value was 0.225 (after 7 iterations) which is an indication of an overall good fit. This is an indication of the degree to which the map represents the data. The pattern matching graph indicated high agreement between the rating variables (what is important versus what is feasible) with a Pearson product-moment correlation of $0.67(r=0.67)$.

\section{Go-zone}

Figure 3 shows the Go-Zone ratings generated for all the 46 statements and the 6-cluster map. Statements rated above the mean for both importance and feasibility are shown in the Go-Zone (the green shaded area of Figure 3). The Go-zone statements were drawn from all the six clusters: 1) Referral Systems; 2) Availability of Equipment; 3) Protocols and Guidelines; 4) Capacity Building/Training; 5) Policy Reform; and 6) Technical Support and Supervision.

\section{Discussion}

Context matters in implementation efforts[30,31]; thus, it is important to understand the key factors that will enhance or limit the effective implementation of evidence-based interventions in resource-constraint settings like Ghana. The main objective of this study was to identify ways to describe national, regional and district health stakeholders' perspectives and characterize the array of strategies needed to enhance the implementation of evidence-based TASSH within Ghana's Community-based Health and Planning Services (CHPS) zones.

Using the GCM, 46 statements grouped under six clusters highlighted 
stakeholder's perceptions of factors likely to influence uptake of TASSH including contextual issues that should be considered and developed with key stakeholders explicitly for the implementation of a task strengthening approach to community-based management of high blood pressure. These findings are relevant particularly in LMICs seeking to implement community-based strategies to ameliorate the burden of high blood pressure.

These six clusters include: 1) Referral Systems; 2) Availability of Equipment; 3) Protocols and Guidelines; 4) Capacity Building/Training; 5) Policy Reform, and 6) Technical Support and Supervision. Cluster 1 highlights how useful a good referral system can facilitate the implementation of the task strengthening strategy to improve hypertension outcomes. Cluster 2 highlights the essence of providing the needed equipment to implement an intervention like this such as the sphygmomanometer, weighing scales as well as height measuring devices. Cluster 3 and 4 while distinct share similar factors for the intervention's implementation such as capacity building, provision of protocols and guidelines suitable for a community-based facility (such as the CHPS zones) to undertake the mandate of screening and referring individuals with hypertension to the next level of care (health centers or district hospitals) for the required treatment. Cluster 5 and 6 also highlight key health systems strengthening strategies that facilitate this community-based approach. The stress value of 0.225 reported by this study lies within the $95 \%$ confidence interval (0.205-0.365) reported by a meta-analysis of concept mapping projects which is an indication that final cluster was a representative of the stakeholders perspectives[20,32].

A pattern match compared importance and feasibility ratings by the cluster. The cluster "availability of equipment" had a high mean rating (mean 4.80 out of 5) for importance followed by "capacity building" as well as both clusters had a high proportion of statements placed within the Go-zone. Also, the cluster "capacity building" received the high mean rating (mean 4.20 out of 5) for feasibility followed by the cluster "availability of equipment". This is an indication that these two strategies were regarded as the most feasible and the most important strategies for the successful implementation of any communitybased hypertension management program. These findings are similar to a study conducted by Blackstone et al., 2017 in the Ashanti Region of Ghana, that found that personnel training was the most feasible and important intervention component [33]. Although important (mean 4.40 out of 5), Policy reform was rated as the least feasible strategy to address. In this study, the overall correlation between the ratings for importance and feasibility was moderately positive $(r=0.67)$. This is an indication that participants opinions on importance aligns with what is considered feasible. The degree of slope of the lines (in Fig. 2) between importance and feasibility demonstrate this alignment. For example, there was much alignment between importance and feasibility to implement indicators within the cluster protocol and guidelines domain. Also, participants agreed on the relative low importance and feasibility of policy reforms as a strategy required for the successful implementation of a community-based hypertension control programme like uptake TASSH.

The statements in the cluster "availability of equipment" offers a rich repository of ideas for identifying the needed logistics and immediate next steps for the successful implementation of a health system strengthening initiatives for hypertension control. Furthermore, statements in the cluster "capacity 
building" highlights the need to develop a capacity building curriculum that includes educational and training materials for the health workers which will be used in the task strengthening training modules. These statements were considered and combined to design the interventions and training package that reflects specific targets and contexts. Although, policy reforms had the least mean rating for both importance and feasibility, the statements in the cluster provides pragmatic implementation strategies that can be implemented within the primary care.

There are two key messages from this formative phase activity. To begin with, for a successful implementation of a community-based hypertension control programme requires multicomponent, multistakeholder action and cooperation. Outputs from this study signals 6 broad areas for interventions as well as specific requirements for action across the various level of care.

Secondly, our work underscores the importance of incorporating the perspectives of healthcare leadership (different stakeholder groups) in highlighting strategies that will be useful for the implementation processes of community-based task-shifting strategies for hypertension management and control. Encouragingly, some of the identified strategies mimic the Ministry of Health strategies sets out to prevent, control and manage non-communicable diseases (NCDs) including hypertension. For example, the policy document as part of its strategic areas of implementation specify health system strengthening; particularly in terms of capacity building and provision of logistics as a priority for preventing and managing NCDs at the community level. [34]

\section{Strength and Limitations}

Key contributions of this study are the identification of key strategies for the implementation of community-based strategies for hypertension control. The stakeholders who participated in this study work at GHS and are quite knowledgeable about the inner works of the health system in Ghana. The understand the capacity for success of an intervention and their numerous years in the field provide a keen insight that will help with the modification of a tasks strengthening intervention. The diverse group of stakeholders who hold positions at the national, regional and district level provide a varied scope of recommendations that can be targeted at each tier of the health system in Ghana when considering the scale of implementation of the intervention in the health system. Statements grouped into clusters are a useful source of information for researchers, policy makers and policy implementing agencies to select and combine to design and implement community-level strategies for hypertension control across different context and various cascade of healthcare. The statements suggested and grouped into clusters provides other implementation strategies which can be considered for future research for its effectiveness, Despite these strengths this study, the team note the following limitations. Although the study sampled and included various stakeholders across different levels of healthcare leadership, the small sample size of 22 may limit the generalizability of the study findings. Albeit this limitation, the study draws on the perspective of key individuals who formulate policy as well as implement policy across the Ghana healthcare system. Therefore, the views shared cut across the various levels of healthcare and are relevant to the existing system. Additionally, we did not include the community health 
officers who were going to implement the task strengthening interventions. However, the participants included leaders of these community health officers which implies that their views may not be different from the community health officers.

\section{Conclusions}

Through an established concept mapping methodology, our study highlights health systems and healthcare leadership perceived factors pertinent to the design and implementation of community-based task-shifting strategies for hypertension management and control. Our findings point to a prioritized set of specific initiatives for the effective implementation of a community-based hypertension programme in Ghana across 6 thematic areas. Furthermore, findings from this study will inform implementers and policymakers as to the best strategies for implementing community-based hypertension interventions in resource-constraint settings to optimize feasibility and acceptability in the local context. Data presented by this study will be useful in chatting a common agenda for a successful implementation of any form of community-based hypertension control programme.

\section{List Of Abbreviations}

\begin{tabular}{|ll|}
\hline CHNs & Community Health Nurses \\
\hline CHPS & Community Health Planning Services \\
\hline CVD & Cardiovascular Disease \\
\hline GCM & Group Concept Mapping \\
\hline HIC & Health Insurance Coverage \\
\hline HTN & Hypertension \\
\hline MDS & Multidimensional Scaling \\
\hline NCDS & Non-Communicable Diseases \\
\hline SD & Standard Deviation \\
\hline SSA & Sub-Saharan Africa \\
\hline TASSH & Task Strengthening Strategy for Hypertension \\
\hline WHO & World Health Organization \\
\hline
\end{tabular}

\section{Declarations}

\section{Ethics approval and consent to participate}


This study was approved by the Ghana Health Service's Ethics Review Committee (GHS-ERC:004/08/17), the Committee on Human Research, Publication and Ethics of the Kwame Nkrumah University of Science and Technology (CHRPE/AP/418/17) and the Institutional Ethics Committee of the Kintampo Health and Research Centre (KHRCIEC/2017-13). Informed consent was obtained from all study participants.

\section{Consent for publication}

Not Applicable

\section{Availability of data and materials}

The data will be made available on request to the corresponding author.

\section{Competing interests}

All authors assert that we do not have any competing interests

\section{Funding}

This study received funding support from the National Heart, Lung, and Blood Institute (NHLBI) of the National Institutes of Health (NIH), award number 1U01HL138638-01 under the Hypertension Outcomes for T4 Research within Lower Middle-Income Countries (Hy-TREC) program. The contents in the manuscript are solely the responsibility of the authors and do not necessarily represent the official views of the NIH.

\section{Authors' contributions}

SN and JI drafted the manuscript. KGAA, KA, JG, KwA coordinated the activities of the study. KGAA, KA, JG, KwA, AA and DO provided a critical review of the initial draft. JI, KP, GO, and JP provided overall guidance as investigators. JI, KP, GO also provided a critical review for this manuscript.

\section{Acknowledgements}

We are grateful to the late Professor Jacob Plange-Rhule for his work ethics, mentorship, and above all his friendship. We would like to thank, Chisom Obiezu-Umeh, MPH, at Saint Louis University for her assistance with the data analysis. The authors are grateful to the National Steering Committee Members, Brong Ahafo Region CHPS zones directors and nurses for their participation in this trial. We are also grateful to the U.S. National Heart, Lung, and Blood Institute of the National Institutes of Health (U01 HL138638) for the funding support.

\section{Consent for publication}

Not Applicable

\section{Competing interests}


The authors declare that they have no competing interest.

\section{References}

1. Kearney PM, Whelton M, Reynolds K, Muntner P, Whelton PK, He J. Global burden of hypertension: Analysis of worldwide data. The lancet. 2005;365(9455):217-23.

2. Labhardt ND, Balo J-R, Ndam M, Grimm J-J, Manga E. Task shifting to non-physician clinicians for integrated management of hypertension and diabetes in rural cameroon: A programme assessment at two years. BMC Health Serv Res. 2010;10(1):339.

3. Mendis S, Johnston SC, Fan W, Oladapo O, Cameron A, Faramawi MF. Cardiovascular risk management and its impact on hypertension control in primary care in low-resource settings: $A$ cluster-randomized trial. Bull World Health Organ. 2010;88:412-9.

4. Barsoum RS. Chronic kidney disease in the developing world. N Engl J Med. 2006;354(10):997-9.

5. Beaglehole R, Epping-Jordan J, Patel V, Chopra M, Ebrahim S, Kidd M, et al. Improving the prevention and management of chronic disease in low-income and middle-income countries: A priority for primary health care. The Lancet. 2008;372(9642):940-9.

6. Hagopian A, Thompson MJ, Fordyce M, Johnson KE, Hart LG. The migration of physicians from subsaharan africa to the united states of america: Measures of the african brain drain. Human resources for health. 2004;2(1):17.

7. Mej'ia A, Pizurki H, Royston E. Physician and nurse migration: Analysis and policy implications, report on a who study. 1979.

8. Pang T, Lansang MA, Haines A. Brain drain and health professionals: A global problem needs global solutions. British Medical Journal Publishing Group; 2002.

9. Unwin N, Setel P, Rashid S, Mugusi F, Mbanya J-C, Kitange H, et al. Noncommunicable diseases in sub-saharan africa: Where do they feature in the health research agenda? Bulletin of the World Health organization. 2001;79:947-53.

10. Mendis S, Abegunde D, Oladapo O, Celletti F, Nordet P. Barriers to management of cardiovascular risk in a low-resource setting using hypertension as an entry point. Journal of hypertension. 2004;22(1):59-64.

11. Anyangwe $S$, Mtonga $C$. Inequities in the global health workforce: The greatest impediment to health in sub-saharan africa. Int J Environ Res Public Health. 2007;4(2):93-100.

12. Asamani JA, Amertil NP, Ismaila $H$, Francis AA, Chebere MM, Nabyonga-Orem J. Nurses and midwives demographic shift in ghana-the policy implications of a looming crisis. Human resources for health. 2019;17(1):1-5.

13. GHS. Human resource directorate annual report for 2017. Accra: Ghana Health Service, Human Resource Directorate; 2018.

14. Gyamfi J, Plange-Rhule J, Iwelunmor J, Lee D, Blackstone SR, Mitchell A, et al. Training nurses in task-shifting strategies for the management and control of hypertension in ghana: A mixed-methods 
study. BMC Health Serv Res. 2017;17(1):1-9.

15. Kweku M, Amu H, Awolu A, Adjuik M, Ayanore MA, Manu E, et al. Community-based health planning and services plus programme in ghana: A qualitative study with stakeholders in two systems learning districts on improving the implementation of primary health care. Plos one. 2020;15(1):e0226808.

16. Nyonator FK, Awoonor-Williams JK, Phillips JF, Jones TC, Miller RA. The ghana community-based health planning and services initiative for scaling up service delivery innovation. Health policy planning. 2005;20(1):25-34.

17. Ogedegbe G, Plange-Rhule J, Gyamfi J, Chaplin W, Ntim M, Apusiga K, et al. Health insurance coverage with or without a nurse-led task shifting strategy for hypertension control: A pragmatic cluster randomized trial in ghana. 2018;15(5):e1002561.

18. O’Neil DS, Lam WC, Nyirangirimana P, Burton WB, Baganizi M, Musominali S, et al. Evaluation of care access and hypertension control in a community health worker driven non-communicable disease programme in rural uganda: The chronic disease in the community project. 2016;31(7):878-83.

19. Aifah A, Onakomaiya D, Iwelunmor J, Oladele D, Gbajabiamila T, Obiezu-Umeh C, et al. Nurses' perceptions on implementing a task-shifting/sharing strategy for hypertension management in patients with hiv in nigeria: A group concept mapping study. 2020;1(1):1-12.

20. Ageberg E, Bunke S, Lucander K, Nilsen P, Donaldson A. Facilitators to support the implementation of injury prevention training in youth handball: A concept mapping approach. Scandinavian journal of medicine science in sports. 2019;29(2):275-85.

21. Cook K, Bergeron KJIJoQM. Using group concept mapping to engage a hard-to-reach population in research: Young adults with life-limiting conditions. 2019;18:1609406919891315.

22. Kane M, Trochim WM. Concept mapping for planning and evaluation. Sage Publications Thousand Oaks, CA; 2007.

23. Burke JG, O'Campo P, Peak GL, Gielen AC, McDonnell KA, Trochim WMJQhr. An introduction to concept mapping as a participatory public health research method. 2005;15(10):1392 - 410.

24. Wutzke S, Roberts N, Willis C, Best A, Wilson A, Trochim W. Setting strategy for system change: Using concept mapping to prioritise national action for chronic disease prevention. Health research policy.

25. systems. 2017;15(1):69.

26. Burke JG, O'Campo P, Peak GL, Gielen AC, McDonnell KA, Trochim WM. An introduction to concept mapping as a participatory public health research method. Qual Health Res. 2005;15(10):1392-410.

27. Ezeanolue EE, Gbadamosi SO, Olawepo JO, Iwelunmor J, Sarpong D, Eze C, et al. An mhealth framework to improve birth outcomes in benue state, nigeria: A study protocol. 2017;6(5):e100.

28. Rosas SR, Kane M. Quality and rigor of the concept mapping methodology: A pooled study analysis. Eval Program Plan. 2012;35(2):236-45.

29. Trochim W, Kane M. Concept mapping: An introduction to structured conceptualization in health care. Int J Qual Health Care. 2005;17(3):187-91. 
30. Noyes-Grosser DM, Rosas SR, Goldman A, Elbaum B, Romanczyk R, Callahan EH. Conceptualizing child and family outcomes of early intervention services for children with asd and their families. Journal of Early Intervention. 2014:1053815114551415.

31. Jacobs SR, Weiner BJ, Bunger AC. Context matters: Measuring implementation climate among individuals and groups. Implementation Science. 2014;9(1):46.

32. Marks R, Clarke AM, O'Sullivan M, Barry MM. Context matters in programme implementation. Health Education. 2010.

33. Rosas SR, Kane M. Quality and rigor of the concept mapping methodology: A pooled study analysis. Evaluation program planning. 2012;35(2):236-45.

34. Blackstone S, Iwelunmor J, Plange-Rhule J, Gyamfi J, Quakyi NK, Ntim M, et al. Sustaining nurse-led task-shifting strategies for hypertension control: A concept mapping study to inform evidence-based practice. Worldviews on Evidence-Based Nursing. 2017;14(5):350-7.

35. Health Mo. National policy for the prevention and control of chronic non-communicable diseases in ghana.; 2012 August 8, 2020.

\section{Figures}

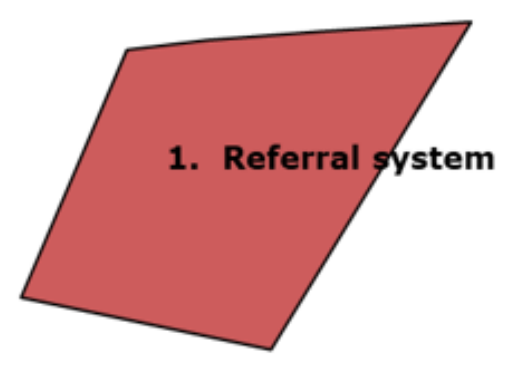

2. Availability of equipment

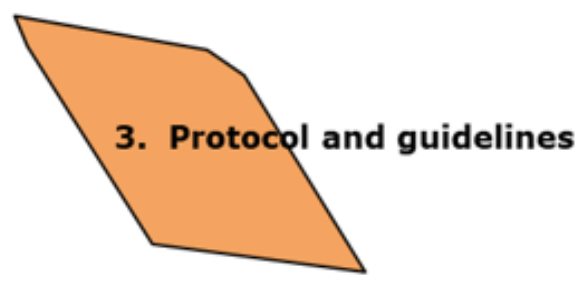

5. Policy roform

4. Capacity building/training

6. Provide technical support and supervision

Figure 1

The six-cluster map of strategies perceived by stakeholders to be useful for the implementation of the TASSH at CHPS zones 


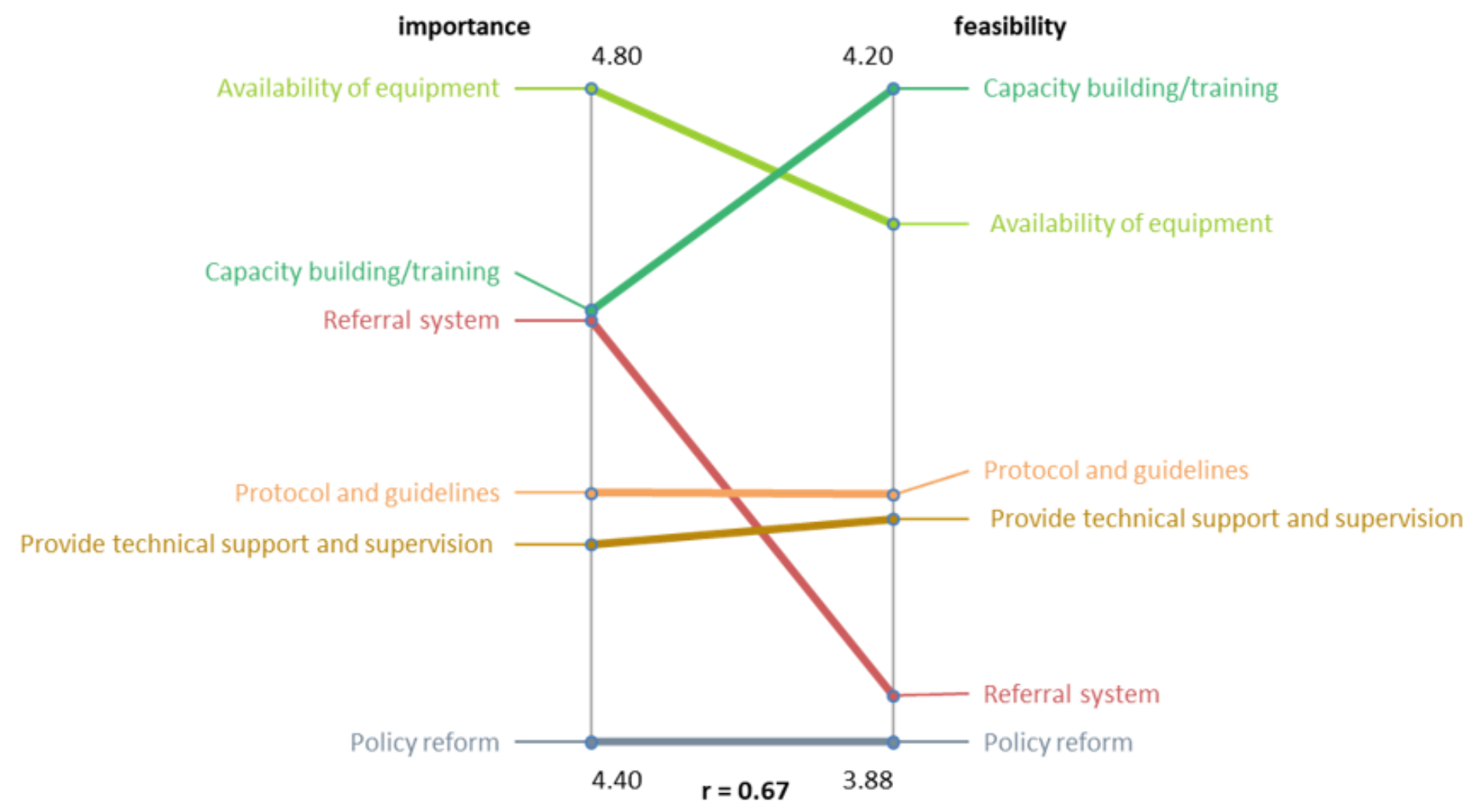

Figure 2

Pattern matching graph for average cluster ratings between importance and feasibility of each cluster 
GO ZONE

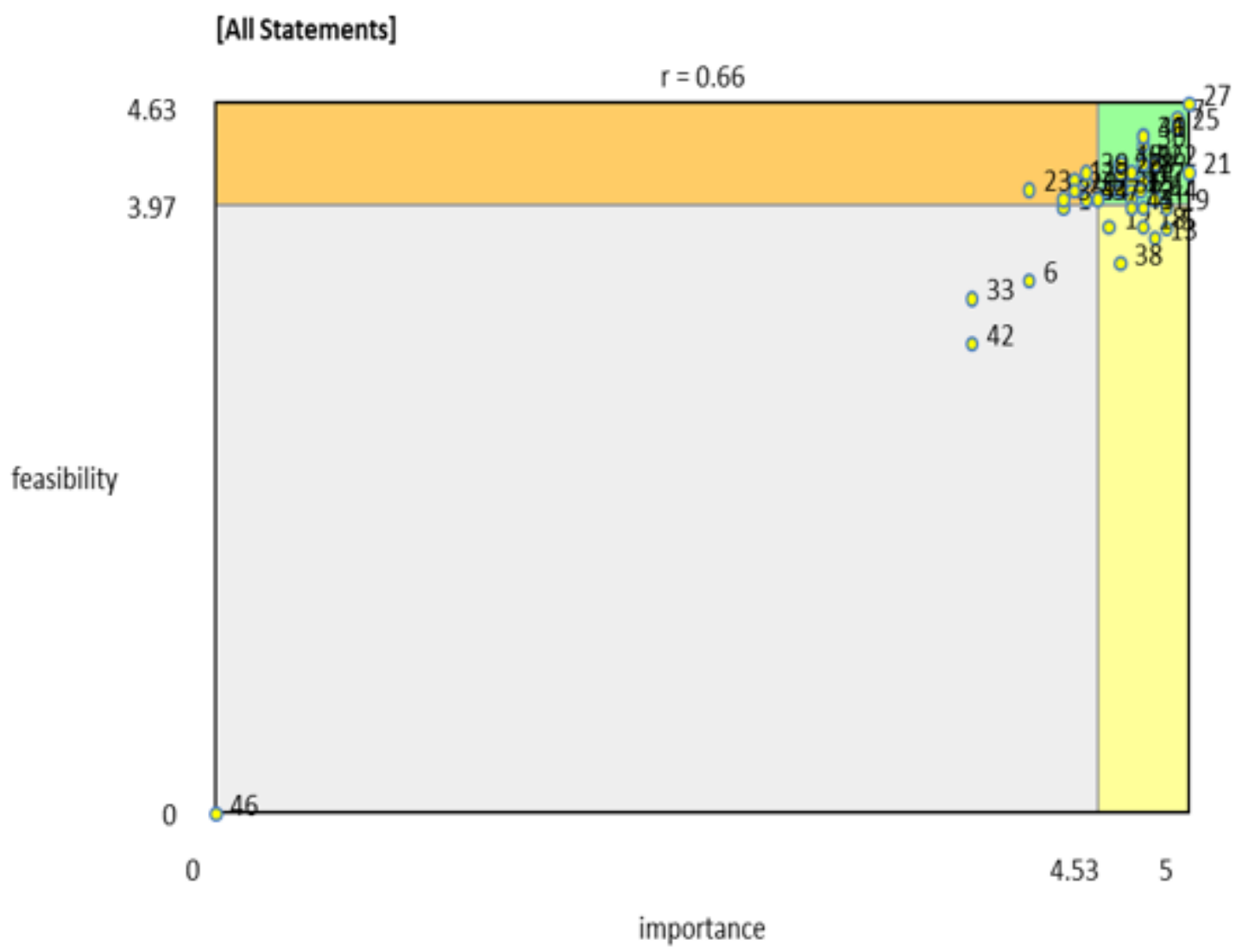

Figure 3

The "Go-Zone" graph showing ratings of importance and feasibility. 